junction of gray and white matter, whereas those arising from contiguous sources are usually superficial and close to the infected bone or dura. During the initial cerebritis (septic encephalitis) stage, the clinical picture is nonspecific. A patient with heart disease develops headache, vomiting, seizures, and fever. As the abscess forms, the neurologic signs become more apparent and lateralizing, with hemiparesis, hemianopia, papilledema, and localized percussion tenderness of the skull. (Raimondi et al. 1965). The EEG shows focal slowing, and CT confirms the diagnosis. In the differential diagnosis, thromboses of arteries, veins and dural sinuses are common in cyanotic infants, and symptoms may mimic an abscess, except the onset is more abrupt. Thromboses are rare in infants older than 2 years. Hypoxic attacks occur in $12 \%$ to $15 \%$ of patients with cyanotic heart disease and are common during the first 2 years of life. Meningitis may also mimic an abscess before symptoms and signs become lateralized. (Menkes JH, 1980). The diagnosis of brain abscess should be considered with new-onset headache and seizure, especially in a child with congenital heart disease or recent sinus or ear infection, commonly Streptococcus milleri ( $S$ intermedius), and in an acutely immunosuppressed patient with fungal disease.

\title{
MRI/MRS STUDY OF ADEM
}

Magnetic resonance imaging and $\mathrm{H}$ magnetic resonance spectroscopy were used to detect possible structural and neurochemical abnormalities in two children, ages 6-months and 4.5 years, with acute disseminated encephalomyelitis at the State University of New York, Stony Brook, New York. The infant presented with focal seizures and thalamic and cerebral white matter lesions, and the older child with tremor and dystonia with bilateral basal ganglia lesions. Both recovered without the use of steroids or IV immunoglobulin. $\mathrm{H}$ MRS of involved areas showed abnormalities in N-acetyl-aspartate, choline, and lactate peaks during the symptomatic phase, and a low $\mathrm{N}$-acetyl-aspartate persisted during recovery. The MRS findings are consistent with neuronal dysfunction, cellular membrane turnover, cellular infiltation, and metabolic stress in the acute phase, and with neuronal loss in the chronic phase. (Gabis LV, Panasci DJ, Andriola MR, Huang W. Pediatr Neurol 2004;30:324-329). (Respond: Dr Lidia V Gabis, Pediatric Neurology, Safra Children's Hospital, Sheba Medical Center, Tel Hashomer, Israel 52621).

COMMENT. Metabolic studies using magnetic resonance spectroscopy should aid in diagnosis of ADEM and may prove of value in following the course of the disease and the need for therapy.

\section{MRS METABOLITES IN RASMUSSEN ENCEPHALITIS}

The evolution of metabolite changes in an 8-year-old boy with focal Rasmussen encephalitis was studied by MRI and MRS at the Brain Research Institute, University of Melbourne, Australia. Serial structural and metabolite changes during a 9-month period which included an episode of complex partial status epilepticus showed focal swelling and a marked increase in T2-weighted signal intensity in the superior temporal gyrus following status. Follow-up scans showed resolution of the swelling and the development of slight focal atrophy. MRS showed a reduction in N-acetylaspartate, total creatine and trimethylamines after status. These metabolite changes had resolved in subsequent MRS 
scans. After surgery, metabolite abnormalities confirmed by MRS suggested active disease in the anterior region of the excised superior temporal gyrus. Rasmussen encephalitis is characterized by a progressive encephalitic process that shows fluctuating evidence of neuronal damage and recovery related to seizures. (Wellard RM, Briellmann RS, Wilson JC et al. Longitudinal study of MRS metabolites in Rasmussen encephalitis. Brain 2004;127:1302-1312). (Respond: Professor Graeme Jackson, Brain Research Center, Austin Health, Neurosciences Building, Banksia St, Heidelberg West 3081, Australia).

COMMENT. Longitudinal studies of MRS metabolites in Rasmussen encephalitis reveal a fluctuating metabolite profile related to seizure activity, indicating the limitations of MRS at a single point in the course of the disease.

\section{RISK OF BRAIN DAMAGE FOLLOWING PERTUSSIS IMMUNIZATION WITH WHOLE-CELL \& ACELLULAR VACCINES}

Serious neurological disorders reported following whole-cell (WC) in comparison to acellular (AC) pertussis vaccines (PV) were evaluated by the Genetic Centers of America, Silver Spring, MD. The Vaccine Adverse Events Reporting System (VAERS) was analyzed for ER visits, hospitalizations, disabilities, deaths, seizures, infantile spasms, encephalitis/encephalopathy, autism, SIDS, and speech disorders reported within 3 days following PV immunization in the US from 1997-1999. Both vaccines were available for use in this time period. The predominant PV in the US and Japan changed from WC to AC in 1998, and WCPV was withdrawn in 2001. The incidence per million of reactions with WCPV/ACPV was for ER visits (72/32), diabilities (1.4/0.38), deaths (2.7/1.5), seizures (13.4/3.6), infantile spasms (0.39/0.11), encephalopathy (0.78/0.095), autism $(0.49 / 0.11)$, SIDS (1.5/0.87), speech disorders $(0.78 / 0.23)$, and cerebellar ataxia $(0.29 / 0.27)$. Statistical increases for all reactions were associated with WCPV, except cerebellar ataxia. WCPV contains 3000 different proteins, including the neurotoxins endotoxin, pertussis toxin, and adenylate cyclase, compared to only 2 to 5 proteins in ACPV and DTaP, which probably account for its greater reactogenicity. (Geier DA, Geier MR. An evaluation of serious neurological disorders following immunization: a comparison of whole-cell pertussis and acellular pertussis vaccines. Brain Dev 2004;26:296-300). (Respond: Mark R Geier, 14 Redgate Ct, Silver Spring, MD 20905).

COMMENT. These results are similar to previous studies conducted by the CDC, using the VAERS database (Braun et al. 2000). Although relatively rare in pediatric practice, serious neurological disorders were undoubtedly linked to the use of whole-cell pertussis vaccine, and since its withrawal from the US market in 2001 and the required substitution of acellular vaccine, the incidence of neurologic adverse events has significantly decreased. Contrary to the frequently proposed viewpoint that post-DPT seizures are coincidental, the earlier reports (Byers, Moll, 1948; et al) of a relationship between seizures, including infantile spasms, encephalopathy and whole-cell PV are supported by the present study. It is unfortunate that the development and substitution of the less neurotoxic acellular PV was delayed for more than 50 years, despite the concerns and reports by pediatric neurologists of a probable causal relationship between seizures and WCPV. 